\title{
Edad y crecimiento del jurel Trachurus murphyi en el Perú
}

\author{
Age and growth of Jack mackerel Trachurus murphyi in Peru
}

Instituto del Mar del Perú, esquina Gamarra y General Valle S/N Chucuito, Callao, Perú.

Email Teobaldo Dioses: tdioses@imarpe.gob.pe
Citación:

Dioses T. Edad y crecimiento del jurel Trachurus murphy en el Perú. En: Csirke J., R. Guevara-Carrasco \& M. Espino (Eds.). Ecología, pesquería y conservación del jurel (Trachurus murphyi) en el Perú. Rev. peru. biol. número especial 20(1): 045- 052 (Septiembre 2013)
Teobaldo Dioses

\section{Resumen}

Se determinó la edad y se estimó el crecimiento del jurel Trachurus murphyi Nichols 1920, mediante la lectura y medición de anillos anuales de crecimiento en 977 pares de otolitos de especímenes entre 3 y $71 \mathrm{~cm}$ de longitud total colectados dentro del dominio marítimo peruano durante el año 1978 y parte de 1977 y 1979. Se comprobó la validez de las marcas anuales en 50 otolitos con 5 marcas o zonas anuales, y también con el análisis del incremento marginal mensual en 240 otolitos de especímenes entre 35 y $39 \mathrm{~cm}$ obtenidos entre julio 1977 y junio 1979. A partir de las medidas de los anillos anuales y sus respectivas tallas retrocalculadas se estimaron los parámetros de crecimiento en longitud y peso de la ecuación de von Bertalanffy: longitud infinita $\left(L_{\infty}\right)=80.77 \mathrm{~cm}$, peso infinito $\left(W_{\infty}\right)=3744.10 \mathrm{~g}$, coeficiente de crecimiento $(k)$ $=0.155 \mathrm{y}$ edad teórica a la longitud $0\left(\mathrm{t}_{0}\right)=-0.356$. Se discuten las diferencias y semejanzas con las curvas y parámetros de crecimiento calculados por otros autores, haciendo énfasis en aquéllos cuyos resultados contribuyen a validar los estimados en el presente trabajo. Según los parámetros estimados, el T. murphyi peruano tendría una velocidad de crecimiento mayor que el T. murphyi de más al sur.

Palabras clave: Edad, crecimiento, otolitos, jurel, Trachurus murphyi.

\section{Abstract}

The age and growth of Jack mackerel Trachurus murphyi Nichols 1920 was determined by the reading and measuring annual growth rings from 977 pairs of otoliths from specimens between 3 and $71 \mathrm{~cm}$ total length collected within the Peruvian maritime domain during 1978 and part of 1977 and 1979. The validity of the annual marks was confirmed with 50 otolith with 5 marks or annual zones, and also with the analysis of the monthly marginal increment in 240 otoliths of specimens between 35 and $39 \mathrm{~cm}$ obtained from July 1977 to June 1979 . From the annual rings measurements and their respective retroestimated sizes, the following parameters of the von Bertalanfy ecuation for growth in length and weight were estimated: infinite length $\left(L_{\infty}\right)=$ $80.77 \mathrm{~cm}$, infinite weight $\left(\mathrm{W}_{\infty}\right)=3744.10 \mathrm{~g}$, growth coefficient $(\mathrm{k})=0.155$, and theoretical age at length $0\left(\mathrm{t}_{0}\right)=-0.356$. The estimated parameters suggest that the Peruvian $T$. murphyi has a faster growth rate than the more southern T. murphyi.

Keywords: Age, growth, otoliths, Jack mackerel, Trachurus murphyi.

\section{Introducción}

El jurel Trachurus murphyi Nichols 1920 es una de las especies pelágicas comerciales más importantes del Pacífico Sudoriental. En Chile alcanzó una captura máxima de más de 4 millones de toneladas en 1994 y en Perú de alrededor de 800 mil toneladas en 2001. Para analizar la dinámica poblacional y evaluar los cambios en la abundancia de este importante recurso pesquero, es de gran utilidad el poder contar con parámetros confiables de edad y crecimiento de la especie a fin de evaluar el estado del stock en el Perú.

Entre los métodos comúnmente aceptados y más empleados en la determinación de la edad y estimación del crecimiento individual, figuran aquellos basados en la lectura de los anillos de crecimiento en otolitos, aplicando diversas metodologías según la especie. En el caso del T. murphyi, en el Perú se han aplicado la lectura tradicional (lectura de anillos de crecimiento en la cara externa de los otolitos enteros), lectura de secciones transversales de otolitos y lectura de anillos diarios. En este trabajo se presentan los resultados de las determinaciones de su edad y crecimiento mediante la lectura tradicional y se comparan con los obtenidos por otros autores con métodos similares.
Publicado online: $\quad 11 / 10 / 2013$ Publicado impreso: $15 / 10 / 2013$ 


\section{Material y métodos}

El material y los respectivos datos analizados en este estudio fueron tomados de los muestreos biológicos efectuados tanto en el laboratorio central del Callao como en los laboratorios costeros de Paita, Chimbote, Pisco e Ilo del Instituto del Mar del Perú (IMARPE). Se trabajó con muestras del año 1978, complementándose para las longitudes extremas con muestras de los años 1977 y 1979. Los muestreos biológicos que realiza el IMARPE corresponden a muestreos sistemáticos estratificados. En el caso de T. murphyi se analizaron mensualmente 10 ejemplares en detalle, por cada centímetro de longitud. De estos ejemplares se obtuvo la siguiente información: longitud total, peso total, longitud y peso de las gónadas, sexo y grado de madurez sexual, contenido de grasa, contenido estomacal. Además se colectan otolitos para la determinación de la edad y gónadas para el seguimiento histológico del desarrollo ovocitario.

En la determinación de la edad de T. murphyi se utilizó el otolito sacular o sagitta. Este otolito cambia gradualmente su apariencia y se observa que toma una forma regular después que ha completado su primer año de vida. Para la lectura de anillos se utilizaron muestras de otolitos provenientes de todo el litoral peruano, tratándose de cubrir el mayor rango posible de tallas presentes en la pesquería. En total se hizo la lectura tradicional en 977 otolitos de otros tantos especímenes machos y hembras de T. murphyi. Para cada otolito se tuvo la medida de longitud total del ejemplar y se agruparon las muestras en rangos de longitud con intervalos de un centímetro. Se contó con ejemplares desde los 3 hasta los $71 \mathrm{~cm}$ de longitud total y, en lo posible, se tomó 15 especímenes por intervalo de longitud. Además, en el análisis del borde de los otolitos y en la determinación del incremento marginal se utilizaron 240 otolitos; mensualmente se tomaron 10 otolitos de especímenes de 35 a $39 \mathrm{~cm}$ de longitud, desde julio de 1977 a junio de 1979.

La metodología de lectura de los anillos de crecimiento es la que utilizaron Misu y Hamasaki (1971) en la merluza peruana (Merluccius gayi peruanus), Samamé (1977) en la sardina (Sardinops sagax), Geldenhuys (1973) en el jurel de Sudáfrica (Trachurus trachurus) y Dioses (1985) en la merluza M. gayi peruanus. Para visualizar los anillos se probaron diferentes técnicas. La que dio mejores resultados fue la de quemar ligeramente la cara interna de los otolitos con un mechero de alcohol, hasta que tomen un color marrón claro. Esto facilitó la observación de las zonas hialinas oscuras así como las opacas un poco blanquecinas.

Los otolitos fueron leídos en una cámara oscura utilizando un proyector de contornos Nikon 6CT-2 a 10 aumentos, utilizando luz reflejada. Las mediciones se efectuaron en milímetros, del núcleo hacia el borde posterior de cada anillo hialino $\left(\mathrm{r}_{\mathrm{n}}\right)$, así como del núcleo hacia el borde posterior o radio del otolito $(\mathrm{R})$, como se puede observar en la Figura 1A. Para ver con mayor nitidez los anillos, el otolito fue colocado en una placa Petri de fondo negro y pincelado con aceite de cedro. Para las lecturas, generalmente se utilizó el otolito derecho, empleándose el izquierdo cuando el primero se encontraba en malas condiciones.

El incremento marginal fue calculado mediante la ecuación:

$$
\mathrm{IM}=\left(\mathrm{R}-\mathrm{r}_{\mathrm{n}}\right) /\left(\mathrm{r}_{\mathrm{n}}-\mathrm{r}_{\mathrm{n}-1}\right)
$$

Donde :

$$
\mathrm{R}=\text { radio del otolito }
$$

$$
\mathrm{r}_{\mathrm{n}}=\text { radio del último anillo formado }
$$

Mediante el método del retrocálculo a edades pretéritas se determinó la longitud que tendría el pez al completar la formación de cada anillo hialino anual. Con este fin se utilizó la ecuación $\mathrm{R}=\mathrm{a}^{\prime} \mathrm{L}^{2}+\mathrm{b}^{\prime} \mathrm{L}+\mathrm{c}^{\prime}$ que relaciona la longitud (L) del pez con la medida del radio $(\mathrm{R})$ del otolito.

Los parámetros de longitud infinita $\left(\mathrm{L}_{\infty}\right)$ y del coeficiente de crecimiento se estimaron por el método gráfico de Ford-Walford (Ford 1933, Walford 1946), para lo cual se plotearon las longitudes medias (L) del T. murphyi a la edad t contra la longitud media a la edad $t+1$, de ello se obtuvo una recta interpolada que corta la bisectriz en un punto correspondiente a la estimación de $\mathrm{L}_{\infty}$. La recta ajustada mediante el método de mínimos cuadrados corresponde a la ecuación de Ford- Walford:

$$
\begin{array}{ll} 
& \mathrm{L}_{\mathrm{t}+1}=\mathrm{a}+\mathrm{bL}_{\mathrm{t}} \\
\text { donde: } & \mathrm{a}=\mathrm{L}_{\infty}\left(1-\mathrm{e}^{-\mathrm{k}}\right) \\
& \mathrm{b}=\mathrm{e}^{-\mathrm{k}} \\
\text { y de donde: } & \mathrm{L}_{\infty}=\mathrm{a} /(1-\mathrm{b}) \\
& \mathrm{k}=-\mathrm{Ln} \mathrm{b}
\end{array}
$$

La edad teórica $\left(\mathrm{t}_{0}\right)$ en la cual el T. murphyi tendría longitud cero se estimó mediante la ecuación de Gulland (1977), que es una derivación de la expresión de crecimiento de von Bertalanffy, según la ecuación:

$$
\mathrm{t}_{0}=\mathrm{t}+(1 / \mathrm{k})^{*} \operatorname{Ln}\left(\left(\mathrm{L}_{\infty}-\mathrm{L}_{\mathrm{t}}\right) / \mathrm{L}_{\infty}\right)
$$

De donde el $t_{0}$ final se calcula como el promedio de los $t_{0}$ obtenidos para las diferentes edades observadas.

\section{Resultados}

\section{Determinación de la edad}

Descripción y medidas de los otolitos.- Esta descripción se hace tomando el otolito de un espécimen de cinco años (Fig. la y b). El otolito tiene una forma un poco lanceolada, moderadamente redondeada en el borde posterior o cauda, mientras que el borde anterior o rostro es alargado y puntiagudo. En el borde dorsal, en dirección al rostro se encuentra el antirrostro, un poco corto y terminado en punta, por encima de una pequeña hendidura. La parte que va del antirrostro hacia el borde ventral es ligeramente lisa y curvada. La cara externa es cóncava, mientras que la interna es convexa y en ella se encuentra el surco acústico, canal que corre en la parte media horizontal y en sentido anteroposterior, terminando en un punto ciego cerca de la cauda.

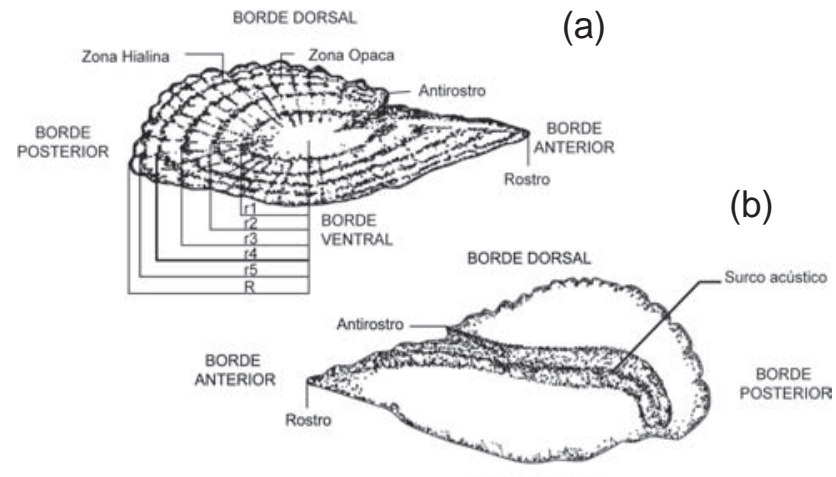

Figura 1. Otolito derecho: a) Cara externa, b) Cara interna Figura 1. Right otolith: a) External surface, b) Internal surface 


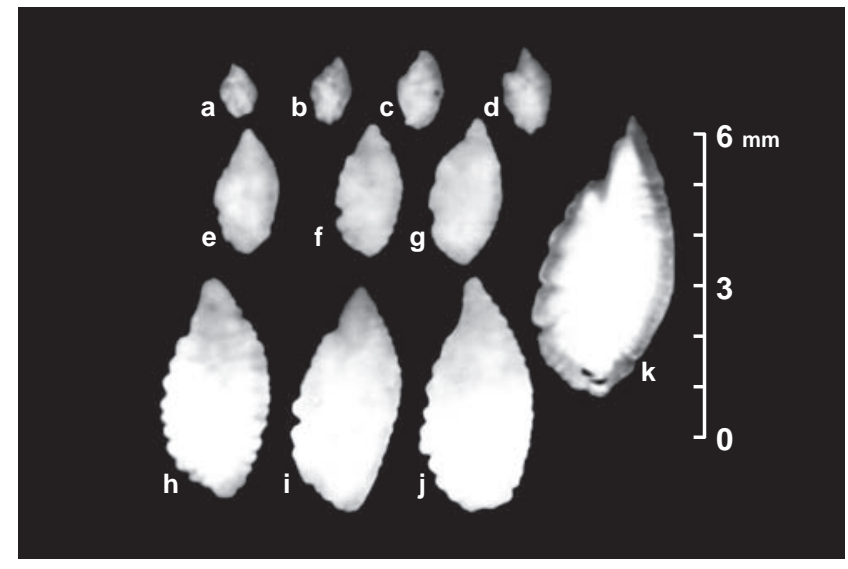

Figura 2. Otolitos mostrando la secuencia de formación del primer anillo anual en jureles T. murphyi de 3 a $17 \mathrm{~cm}$ de longitud total (LT): a) $3 \mathrm{~cm}$, b) $4 \mathrm{~cm}$, c) $5 \mathrm{~cm}$, d) $6 \mathrm{~cm}$, e) $7 \mathrm{~cm}$, f) $8 \mathrm{~cm}, \mathrm{~g}) 9 \mathrm{~cm}$, h) 13 $\mathrm{cm}$, i) $14 \mathrm{~cm}$, j) $15 \mathrm{~cm} \mathrm{y} \mathrm{k)} 17 \mathrm{~cm}$.

Figura 2. Otoliths showing the evolution in the formation of the first annual ring in Jack mackerels T. murphyi from 3 to 17 total length (TL): a) $3 \mathrm{~cm}$, b) $4 \mathrm{~cm}$, c) $5 \mathrm{~cm}$, d) $6 \mathrm{~cm}$, e) $7 \mathrm{~cm}$, f) $8 \mathrm{~cm}, \mathrm{~g}) 9 \mathrm{~cm}, \mathrm{~h}$ ) $13 \mathrm{~cm}$, i) $14 \mathrm{~cm}$, j) $15 \mathrm{~cm}$ and k) $17 \mathrm{~cm}$.

En la cara externa del otolito, usando luz reflejada, se observa una disposición concéntrica de anillos o zonas opacas (blanquecinas) y hialinas (traslúcidas) que se forman alrededor de un núcleo central o foco. Para la interpretación de la edad se utilizaron los anillos hialinos, por ser éstos más nítidos, considerándose en estos estudios a un anillo opaco más un anillo hialino como una zona anual.

Se observa que durante la formación del primer anillo anual hialino (Fig. 2) se desarrolla una mayor continuidad en el entorno del otolito a medida que alcanza un determinado grosor. Un criterio para determinar los siguientes anillos anuales y adjudicarlos como tales fue que éstos debían tener continuidad en la mayor parte del otolito, además de tener un grosor similar o equivalente al grosor observado en el primer anillo anual (Figs. 3, 4, 5 y 6). Por ejemplo, en la Figura 3, entre el primer y segundo anillo anual se puede observar la presencia de dos anillos que se descartaron al ser considerados como anillos falsos, por no satisfacer los criterios indicados.

Se comprobó la validez de esta metodología a través de la comparación con dos aspectos del crecimiento de los otolitos como son la similitud de crecimiento entre la medida de las marcas (cuyo crecimiento decrece con la formación de una nueva marca) y la variación mensual del incremento marginal.

\section{Similitud entre las medidas de las marcas de los otolitos.-} Se usaron 50 otolitos con 5 marcas o zonas anuales (anillos hialinos) y se relacionó la medida de cada marca $\left(r_{n}\right)$ con la longitud o radio (R) del otolito. En la Figura 7 se pueden apreciar las líneas de regresión para cada marca en relación a la longitud del otolito. Se hace notar la evidente similitud de formación entre las marcas de este grupo de otolitos, cuyo crecimiento decrece con la formación de una nueva marca.

Variación del incremento marginal.- Para analizar la periodicidad de variación de los anillos anuales de los otolitos se midió el incremento marginal de especímenes cuyas longitudes oscilaron entre 35 y $39 \mathrm{~cm}$, para cada mes entre julio de 1977 y junio de 1979. En la Figura 8 se puede apreciar que a partir del mes de junio hasta setiembre de 1978 (periodo invernal) se

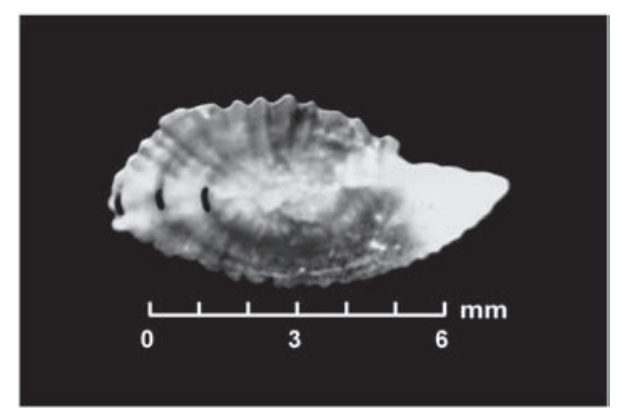

Figura 3. Otolito de edad 3+ años de un jurel T. murphyi de $33 \mathrm{~cm} \mathrm{LT}$. Figure 3. Age 3+ years otolith of Jack mackerel T.murphyi of $33 \mathrm{~cm} \mathrm{TL}$

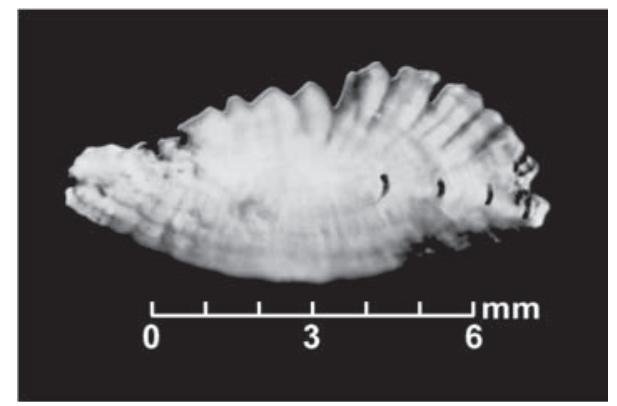

Figura 4. Otolito de edad 4+ años de un jurel T. murphyi de $42 \mathrm{~cm} \mathrm{LT}$. Figure 4. Age 4+ years otolith of Jack mackerel T. murphyi of $42 \mathrm{~cm}$ TL.

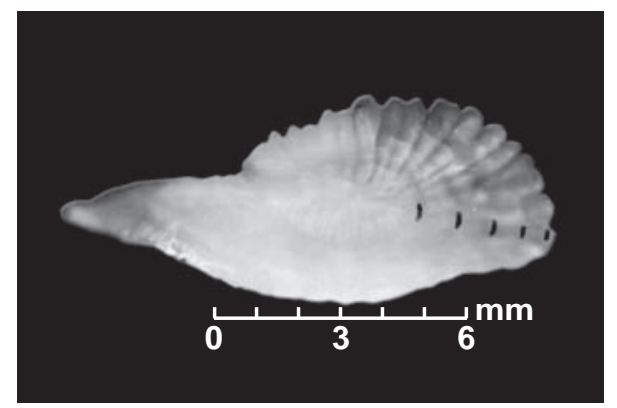

Figura 5. Otolito de edad 5+ años de un jurel T. murphyi de $54 \mathrm{~cm} \mathrm{LT}$. Figure 5. Age 5+ years otolith of Jack mackerel T. murphyi of $54 \mathrm{~cm}$ TL.

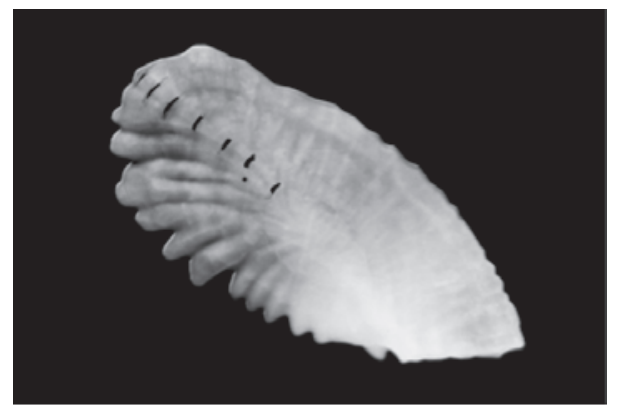

Figura 6. Otolito de edad 7+ de un jurel T. murphyi de $63 \mathrm{~cm} \mathrm{LT}$. Figure 6. Age 7+ years otolith of Jack mackerel T. murphyi of $63 \mathrm{~cm}$ TL.

presentan los valores mínimos, lo que estaría indicando el final de la formación de un anillo y el inicio de la formación de un nuevo anillo hialino. De esta observación podemos concluir que la formación de una nueva zona de crecimiento se inicia en los meses de primavera, lo que se evidencia por el aumento del incremento marginal. Igualmente, para el año 1977 los menores valores se observan en los meses de invierno, especialmente en agosto y setiembre. Para el año 1979, en el mes de junio ya se observó cierta tendencia a la disminución de este incremento luego de haber llegado al máximo valor en abril de ese mismo año. 


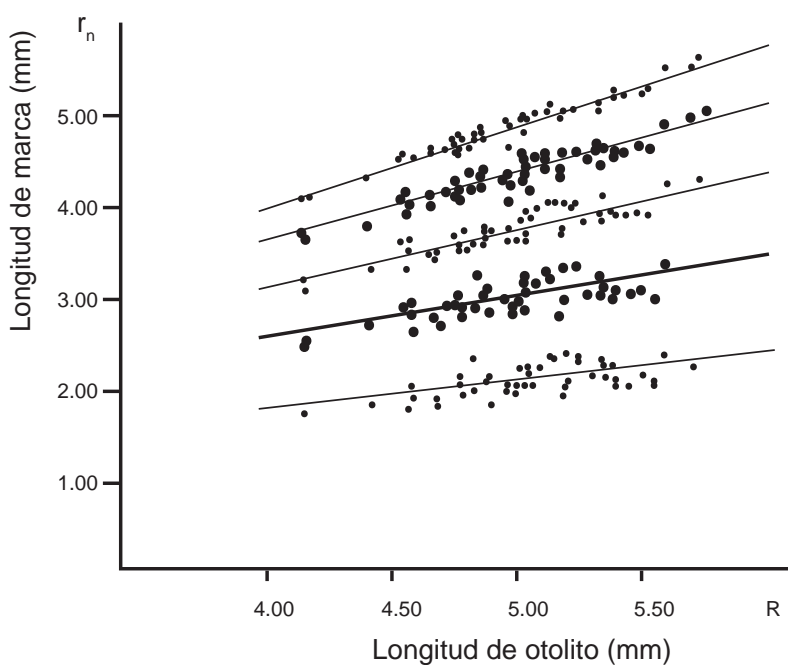

Figura 7. Líneas de regresión entre la medida total o radio del otolito $(R)$ y la medida de los radios de las marcas anuales $\left(r_{n}\right)$ para especímenes con 5 anillos.

Figura 7. Regression lines of the relationship between otoliths radius $(R)$ and the radius of annual rings $\left(r_{n}\right)$ for individuals with 5 rings.

Fluctuación de las zonas hialinas y opacas en el borde de los otolitos.- Se realizó un análisis de la formación periódica de los bordes del otolito a través de la observación de la distribución de material hialino y opaco acumulado mensualmente en el borde de los otolitos entre julio 1977 y junio 1979. Se determinó que el aumento de la frecuencia de bordes opacos se produjo durante el verano, que coincide con el periodo de mayor crecimiento del pez y que es seguido por el aumento de la frecuencia de bordes hialinos durante el invierno, cuando el crecimiento del pez es menor. Estas fluctuaciones confirman lo observado en el incremento marginal. El mayor porcentaje de bordes hialinos se presenta en los meses de invierno, en agosto del año 1977 y en los meses de julio y setiembre en 1978. El porcentaje de bordes hialinos disminuye a medida que los bordes opacos aumentan. Estos bordes opacos alcanzan su máxima frecuencia en los meses de febrero, marzo y abril en el año 1977 y en enero, febrero y marzo en el año 1979.

De lo observado se deduce que existe una formación cíclica de los bordes hialinos y opacos. Esta periodicidad corresponde

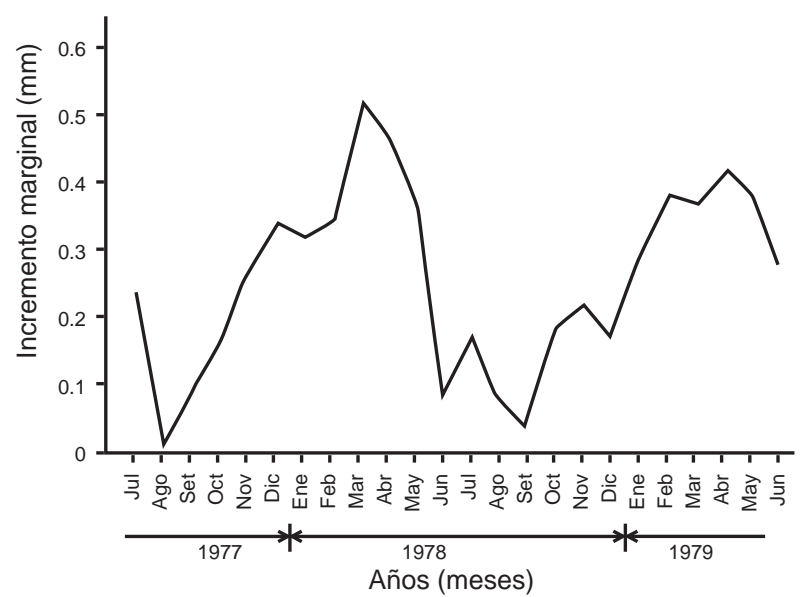

Figura 8. Variación mensual media del incremento marginal de los otolitos de jurel T. murphyi periodo julio 1977 - junio 1979.

Figure 8. Monthly variation of the marginal increment of Jack mackerel T. murphyi otoliths, for the period July 1977 - June 1979. a la formación de un anillo anual durante los meses de mayor intensidad del desove de esta especie, lo que nos permite sugerir el $1^{\circ}$ de enero como fecha de nacimiento arbitraria biológica, para la elaboración de las claves edad-longitud.

\section{Determinación del crecimiento}

Relación entre la longitud del pez y el radio total (R) del otolito.- La medida promedio de la distancia núcleo-borde posterior de los 977 otolitos (R) se relacionó con las respectivas longitudes totales (L) de los peces, según los valores de la Tabla 1. Al correlacionar estas variables, la regresión que mejor se ajustó a las observaciones es la de tipo cuadrática, que responde a la expresión:

$$
\mathrm{R}=-0.0008798 \mathrm{~L}^{2}+0.141083 \mathrm{~L}+0.283965
$$

El ajuste y datos observados están representados gráficamente en la Figura 9.

Cálculo de los parámetros de crecimiento.- En el material observado se encontró otolitos hasta con 11 marcas. En la Tabla 2 se dan las medidas promedios de los radios de los otolitos por cada grupo de marca. Las longitudes promedio del pez a cada edad se determinaron por el método del retro-cálculo. La Tabla 3 presenta las longitudes resultantes de la sustitución de $r_{n}$, por $R$ de la ecuación anterior. Las longitudes promedio $\left(\mathrm{L}_{\mathrm{n}}\right)$ obtenidas y presentadas en la Tabla 3 para cada marca anual serán utilizadas más adelante en el cálculo de la longitud asintótica $\left(\mathrm{L}_{\infty}\right)$ y el parámetro de crecimiento $\mathrm{k}$..

Ploteo de Ford-Walford.- El ploteo de Ford-Walford se puede observar en la Figura 10 y la ecuación resultante de este ploteo es la siguiente:

$$
\mathrm{L}_{(\mathrm{t}+1)}=11.6208+0.8561 \mathrm{~L}_{\mathrm{t}}
$$

Tabla 1. Valores de longitud total, radio del otolito $(R)$ y frecuencias (f) utilizados en la determinación de la edad del jurel T. murphyi.

Table 1. Total length, otolith radius $(R)$ and frequency ( $f$ ) of specimens

\begin{tabular}{|c|c|c|c|c|c|c|c|c|}
\hline $\begin{array}{l}\text { Long. } \\
\text { total } \\
(\mathrm{cm})\end{array}$ & $\mathbf{f}$ & $\underset{(\mathrm{mm})}{\mathrm{R}}$ & $\begin{array}{l}\text { Long } \\
\text { total } \\
\text { (cm) }\end{array}$ & f & $\underset{(\mathrm{mm})}{\mathrm{R}}$ & $\begin{array}{l}\text { Long. } \\
\text { total } \\
(\mathrm{cm})\end{array}$ & $\mathbf{f}$ & $\underset{(\mathrm{mm})}{\mathrm{R}}$ \\
\hline 3 & 3 & 0.53 & 26 & 15 & 3.45 & 49 & 15 & 5.01 \\
\hline 4 & 15 & 0.71 & 27 & 15 & 3.47 & 50 & 15 & 4.98 \\
\hline 5 & 15 & 0.82 & 28 & 15 & 3.55 & 51 & 15 & 5.08 \\
\hline 6 & 15 & 0.97 & 29 & 15 & 3.61 & 52 & 15 & 5.14 \\
\hline 7 & 15 & 1.11 & 30 & 15 & 3.74 & 53 & 15 & 5.20 \\
\hline 8 & 15 & 1.22 & 31 & 15 & 3.93 & 54 & 15 & 5.26 \\
\hline 9 & 15 & 1.38 & 32 & 15 & 3.98 & 55 & 15 & 5.28 \\
\hline 10 & 15 & 1.58 & 33 & 15 & 4.07 & 56 & 15 & 5.38 \\
\hline 11 & 15 & 1.68 & 34 & 15 & 4.14 & 57 & 15 & 5.38 \\
\hline 12 & 15 & 1.81 & 35 & 15 & 4.15 & 58 & 15 & 5.47 \\
\hline 13 & 15 & 1.97 & 36 & 15 & 4.28 & 59 & 15 & 5.52 \\
\hline 14 & 15 & 2.16 & 37 & 15 & 4.26 & 60 & 15 & 5.52 \\
\hline 15 & 15 & 2.32 & 38 & 15 & 4.43 & 61 & 15 & 5.59 \\
\hline 16 & 15 & 2.42 & 39 & 15 & 4.45 & 62 & 15 & 5.75 \\
\hline 17 & 15 & 2.57 & 40 & 15 & 4.51 & 63 & 15 & 5.72 \\
\hline 18 & 15 & 2.72 & 41 & 15 & 4.49 & 64 & 15 & 5.73 \\
\hline 19 & 15 & 2.82 & 42 & 15 & 4.58 & 65 & 15 & 5.95 \\
\hline 20 & 15 & 2.85 & 43 & 15 & 4.63 & 66 & 15 & 5.94 \\
\hline 21 & 15 & 2.97 & 44 & 15 & 4.68 & 67 & 15 & 5.98 \\
\hline 22 & 15 & 3.04 & 45 & 15 & 4.72 & 68 & 7 & 5.96 \\
\hline 23 & 14 & 3.19 & 46 & 15 & 4.78 & 69 & 8 & 6.02 \\
\hline 24 & 10 & 3.25 & 47 & 15 & 4.90 & 70 & 3 & 6.03 \\
\hline 25 & 15 & 3.36 & 48 & 15 & 4.96 & 71 & 2 & 6.10 \\
\hline
\end{tabular}
used to determine the age of Jack mackerel T. murphyi. 


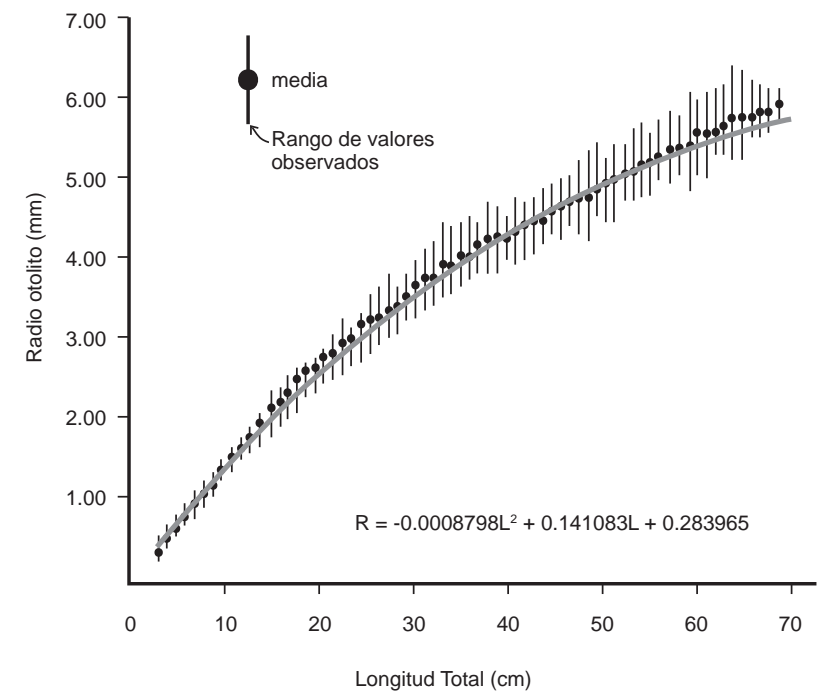

Figura 9. Regresión cuadrática entre la longitud total y el radio del otolito de jurel T. murphyi.

Figure 9. Quadratic regression between total length and otolith radius of Jack mackerel T. murphyi.

Parámetros de crecimiento en longitud.- Como resultado de estos análisis y el ajuste de las ecuaciones indicadas se estimaron los siguientes parámetros de crecimiento para el jurel: $\mathrm{L}_{\infty}=80.77 \mathrm{~cm}, \mathrm{k}=0.155 \mathrm{y} \mathrm{t}_{\mathrm{o}}=-0.356$. La curva de crecimiento teórico resultante (línea continua) y los valores observados de longitud media a la edad (puntos) aparecen representados gráficamente en la Figura 11, donde es de destacar la correspondencia entre los puntos de las longitudes promedio observadas y los valores de la curva de crecimiento teórico calculada.

Parámetros de crecimiento en peso.- Primero se determinó la relación longitud-peso del T. murphyi con datos de 1654 especímenes, cuyas longitudes variaron entre 3.0 y $71.0 \mathrm{~cm}$, que incluyeron 27 ejemplares por rango de longitud (Tabla 4). Con estos valores se ajustó con un $\mathrm{r}=0,9984$ la ecuación longitudpeso $\mathrm{W}=0.0137 \mathrm{~L}_{\mathrm{t}}^{2.8}$ que se presenta gráficamente en la Figura 12 junto a los valores observados. En esta ecuación se destaca el exponente (2.85) que al ser diferente de 3 está indicando que el T. murphyi tiene un crecimiento alométrico. Según estos estimados, la ecuación de crecimiento en peso según el modelo de von Bertalanffy fue la siguiente:

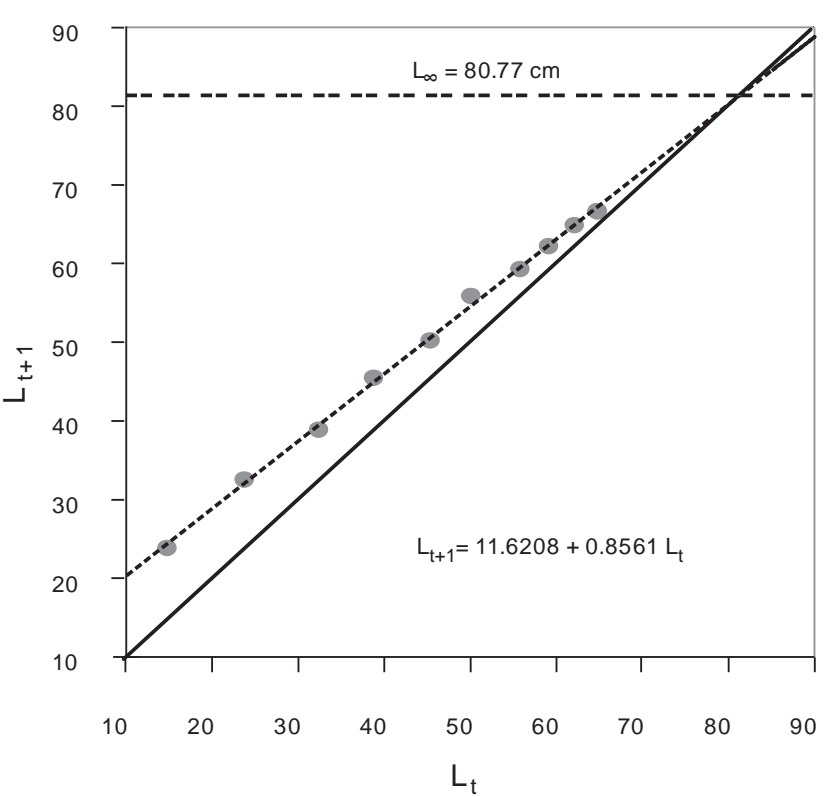

Figura 10 . Ploteo de Ford - Walford.

Figure 10. Ford-Walford Plot.

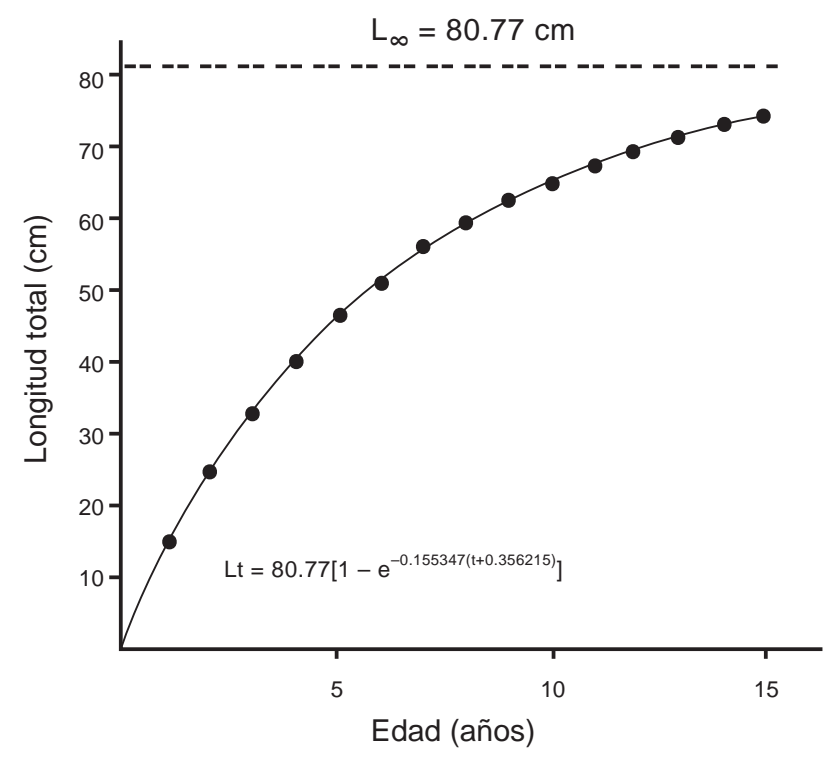

Figura 11. Crecimiento en longitud del jurel según von Bertalanffy. Figure 11. von Bertalanffy growth in length of Jack mackerel.

Tabla 2. Frecuencia (f) y medidas promedio de los radios ( $\mathrm{r}$, en $\mathrm{mm}$ ) de las marcas de crecimiento en otolitos de jurel $T$. murphyi.

Table 2. Frequency (f) and mean radius ( $r$, in $\mathrm{mm}$ ) of the growth rings in Jack mackerel $T$. murphy otoliths.

\begin{tabular}{|c|c|c|c|c|c|c|c|c|c|c|c|c|}
\hline Grupo/Edad & F & r1 & r2 & $\mathrm{r} 3$ & r4 & r5 & r6 & $\mathbf{r} 7$ & r8 & r9 & r10 & r11 \\
\hline 0 & 155 & & & & & & & & & & & \\
\hline 1 & 123 & 2.2 & & & & & & & & & & \\
\hline 2 & 119 & 2.18 & 3.14 & & & & & & & & & \\
\hline 3 & 143 & 2.16 & 3.13 & 3.96 & & & & & & & & \\
\hline 4 & 127 & 2.16 & 3.12 & 3.88 & 4.43 & & & & & & & \\
\hline 5 & 111 & 2.2 & 3.15 & 3.95 & 4.44 & 4.9 & & & & & & \\
\hline 6 & 70 & 2.18 & 3.16 & 3.94 & 4.45 & 4.87 & 5.16 & & & & & \\
\hline 7 & 55 & 2.17 & 3.14 & 3.95 & 4.42 & 4.88 & 5.14 & 5.44 & & & & \\
\hline 8 & 38 & 2.2 & 3.15 & 3.93 & 4.43 & 4.87 & 5.15 & 5.4 & 5.57 & & & \\
\hline 9 & 21 & 2.18 & 3.15 & 3.94 & 4.42 & 4.86 & 5.14 & 5.42 & 5.55 & 5.67 & & \\
\hline 10 & 8 & 2.18 & 3.13 & 3.95 & 4.44 & 4.85 & 5.16 & 5.44 & 5.54 & 5.65 & 5.73 & \\
\hline 11 & 7 & 2.2 & 3.14 & 3.92 & 4.45 & 4.9 & 5.14 & 5.4 & 5.56 & 5.65 & 5.74 & 5.78 \\
\hline Frecuencia total & 977 & 822 & 699 & 580 & 437 & 310 & 199 & 129 & 74 & 36 & 15 & 7 \\
\hline Media de los radios (r) & & 2.18 & 3.14 & 3.94 & 4.44 & 4.88 & 5.15 & 5.42 & 5.56 & 5.66 & 5.74 & 5.78 \\
\hline
\end{tabular}


Tabla 3. Frecuencia (f) y longitudes medias (L) de jurel T. murphyi calculadas de las medias de los radios de los otolitos.

Table 3. Frequency (f) and mean length $(L)$ of Jack mackerel $T$. murpyi calculated from average otoliths radius.

\begin{tabular}{|c|c|c|c|c|c|c|c|c|c|c|c|c|}
\hline Grupo/Edad & $\mathrm{f}$ & r1 & r2 & r3 & $\mathrm{r} 4$ & r5 & r6 & $\mathrm{r} 7$ & r8 & r9 & r10 & r11 \\
\hline 1 & 123 & 14.99 & & & & & & & & & & \\
\hline 2 & 119 & 14.81 & 23.77 & & & & & & & & & \\
\hline 3 & 143 & 14.64 & 23.67 & 32.74 & & & & & & & & \\
\hline 4 & 127 & 14.64 & 23.57 & 31.79 & 38.75 & & & & & & & \\
\hline 5 & 111 & 14.99 & 23.87 & 32.62 & 38.88 & 45.78 & & & & & & \\
\hline 6 & 70 & 14.81 & 23.97 & 32.5 & 39.02 & 45.29 & 50.38 & & & & & \\
\hline 7 & 55 & 14.73 & 23.77 & 32.62 & 38.61 & 45.46 & 50 & 56.3 & & & & \\
\hline 8 & 38 & 14.99 & 23.87 & 32.38 & 38.75 & 45.29 & 50.19 & 55.37 & 59.62 & & & \\
\hline 9 & 21 & 14.9 & 23.87 & 32.5 & 38.61 & 45.13 & 50 & 55.83 & 59.07 & 62.59 & & \\
\hline 10 & 8 & 14.81 & 23.67 & 32.62 & 38.88 & 44.97 & 50.38 & 56.3 & 58.81 & 61.95 & 64.64 & \\
\hline 11 & 7 & 14.99 & 23.77 & 32.26 & 39.02 & 45.78 & 50 & 55.37 & 59.34 & 61.95 & 65.01 & 66.58 \\
\hline Frecuencia total & 822 & 822 & 699 & 580 & 437 & 310 & 199 & 129 & 74 & 36 & 15 & 7 \\
\hline Longitudes medias & & 14.85 & 23.78 & 32.45 & 38.82 & 45.39 & 50.16 & 55.83 & 59.21 & 62.16 & 64.83 & 66.58 \\
\hline
\end{tabular}

Tabla 4. Longitud total, peso (W) y frecuencias (f) de los especímenes de jurel T. murphyi utilizados en la determinación de la relación longitud-peso.

Table 4. Total length, weight (W) and frequency (f) of the specimens of Jack mackerel T. murphyi used in the length-weigh relationship.

\begin{tabular}{ccccccccc}
\hline $\begin{array}{c}\text { Long. } \\
\text { total } \\
\mathbf{( c m )}\end{array}$ & $\mathbf{f}$ & $\mathbf{W} \mathbf{( g )}$ & $\begin{array}{c}\text { Long. } \\
\text { total } \\
\mathbf{( c m})\end{array}$ & $\mathbf{f}$ & $\mathbf{W} \mathbf{( g )}$ & $\begin{array}{c}\text { Long. } \\
\text { total } \\
\mathbf{( c m})\end{array}$ & $\mathbf{f}$ & $\mathbf{W}(\mathbf{g})$ \\
\hline $\mathbf{3}$ & 4 & 0.31 & $\mathbf{2 6}$ & 27 & 142.85 & $\mathbf{4 9}$ & 27 & 901.59 \\
$\mathbf{4}$ & 6 & 0.77 & $\mathbf{2 7}$ & 27 & 159.67 & $\mathbf{5 0}$ & 27 & 966.44 \\
$\mathbf{5}$ & 20 & 1.45 & $\mathbf{2 8}$ & 27 & 173.48 & $\mathbf{5 1}$ & 27 & 985.40 \\
$\mathbf{6}$ & 20 & 2.17 & $\mathbf{2 9}$ & 27 & 196.00 & $\mathbf{5 2}$ & 27 & 1065.59 \\
$\mathbf{7}$ & 21 & 3.52 & $\mathbf{3 0}$ & 27 & 225.33 & $\mathbf{5 3}$ & 27 & 1106.37 \\
$\mathbf{8}$ & 25 & 5.02 & $\mathbf{3 1}$ & 27 & 244.96 & $\mathbf{5 4}$ & 27 & 1161.85 \\
$\mathbf{9}$ & 27 & 7.07 & $\mathbf{3 2}$ & 27 & 251.37 & $\mathbf{5 5}$ & 27 & 1276.00 \\
$\mathbf{1 0}$ & 27 & 10.27 & $\mathbf{3 3}$ & 27 & 283.00 & $\mathbf{5 6}$ & 27 & 1385.62 \\
$\mathbf{1 1}$ & 27 & 13.14 & $\mathbf{3 4}$ & 27 & 311.60 & $\mathbf{5 7}$ & 27 & 1413.93 \\
$\mathbf{1 2}$ & 27 & 16.97 & $\mathbf{3 5}$ & 27 & 332.85 & $\mathbf{5 8}$ & 27 & 1465.22 \\
$\mathbf{1 3}$ & 27 & 21.37 & $\mathbf{3 6}$ & 27 & 348.11 & $\mathbf{5 9}$ & 27 & 1568.81 \\
$\mathbf{1 4}$ & 27 & 26.67 & $\mathbf{3 7}$ & 27 & 377.93 & $\mathbf{6 0}$ & 27 & 1685.44 \\
$\mathbf{1 5}$ & 27 & 34.60 & $\mathbf{3 8}$ & 27 & 398.44 & $\mathbf{6 1}$ & 27 & 1677.74 \\
$\mathbf{1 6}$ & 27 & 40.66 & $\mathbf{3 9}$ & 27 & 432.15 & $\mathbf{6 2}$ & 27 & 1780.96 \\
$\mathbf{1 7}$ & 27 & 49.27 & $\mathbf{4 0}$ & 27 & 479.96 & $\mathbf{6 3}$ & 27 & 1933.85 \\
$\mathbf{1 8}$ & 27 & 59.29 & $\mathbf{4 1}$ & 27 & 514.89 & $\mathbf{6 4}$ & 27 & 2081.50 \\
$\mathbf{1 9}$ & 23 & 57.17 & $\mathbf{4 2}$ & 27 & 585.48 & $\mathbf{6 5}$ & 27 & 2162.16 \\
$\mathbf{2 0}$ & 27 & 66.22 & $\mathbf{4 3}$ & 27 & 589.52 & $\mathbf{6 6}$ & 27 & 2245.04 \\
$\mathbf{2 1}$ & 27 & 79.81 & $\mathbf{4 4}$ & 27 & 662.59 & $\mathbf{6 7}$ & 27 & 2465.38 \\
$\mathbf{2 2}$ & 14 & 93.21 & $\mathbf{4 5}$ & 27 & 688.78 & $\mathbf{6 8}$ & 9 & 2485.11 \\
$\mathbf{2 3}$ & 9 & 104.67 & $\mathbf{4 6}$ & 27 & 743.44 & $\mathbf{6 9}$ & 12 & 2550.92 \\
$\mathbf{2 4}$ & 10 & 107.50 & $\mathbf{4 7}$ & 27 & 821.93 & $\mathbf{7 0}$ & 4 & 2644.50 \\
$\mathbf{2 5}$ & 27 & 125.92 & $\mathbf{4 8}$ & 27 & 855.07 & $\mathbf{7 1}$ & 2 & 2785.00 \\
\hline & & & & & & & &
\end{tabular}

$$
\mathrm{W}_{\mathrm{t}}=3744.10\left[1-\mathrm{e}^{\left(-0.1553^{*}(\mathrm{t}+0.3562)\right)}\right]^{2.84919}
$$

\section{Discusión}

En los estudios de la edad y el crecimiento del jurel T. murphyi en el Pacífico Suroriental se han venido aplicando tres técnicas de preparación e interpretación de los anillos de crecimiento presentes en los otolitos sagita. Dichas técnicas son las siguientes: (a) la lectura tradicional (rutinaria) de anillos anuales en otolitos enteros, (b) la lectura de anillos anuales en cortes transversales de los otolitos y (c) la lectura de microincrementos o anillos diarios.

$\mathrm{Al}$ analizar los resultados de la aplicación de estas técnicas en la lectura de otolitos de jurel T. murphyi en la región se observa que

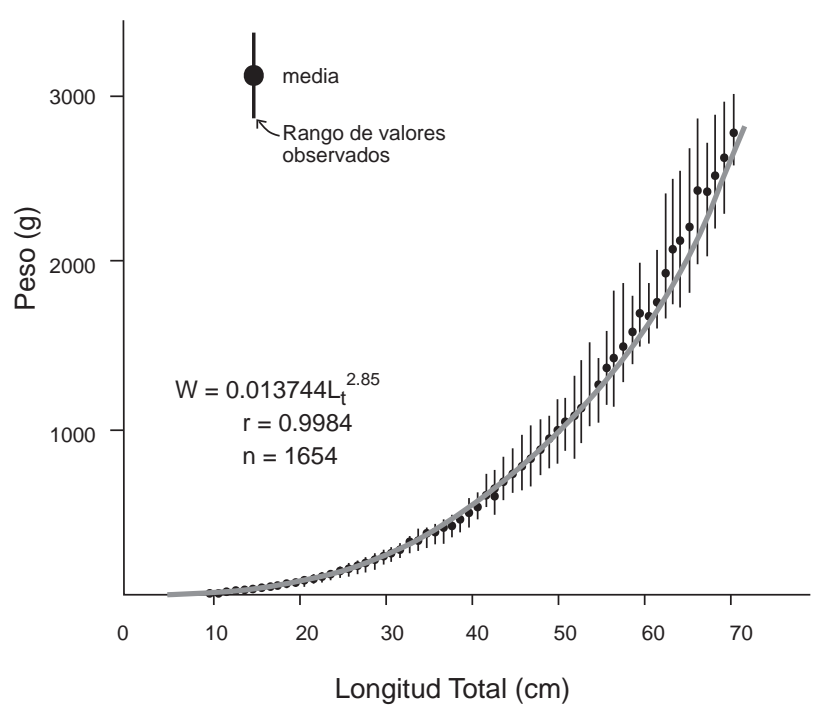

Figura 12. Relación longitud total - peso del jurel T. murphyi.

Figure 12. Weight - length relationship for Jack mackerel T. murphyi.

existen diferencias en las determinaciones de la edad y en los estimados de los parámetros de crecimiento. Algunas de estas diferencias podrían deberse a las diversas técnicas de preparación y dificultades en la aplicación de criterios de interpretación y separación de los anillos anuales o a distinto instrumental óptico empleado. Sin embargo, es evidente que estas diferencias reflejan diversidad de velocidades de crecimiento expresadas en sus correspondientes tasas o coeficientes $(\mathrm{k})$ y, asimismo, en las tallas máximas de crecimiento $\left(\mathrm{L}_{\infty}\right)$ del T. murphyi proveniente de zonas y latitudes distintas o de stocks o substocks más o menos independientes.

Para fines comparativos, en la Tabla 5 se presentan los parámetros de crecimiento de T. murphyi proveniente de distintas zonas del Pacífico Sudoriental que han sido estimados por diversos autores siguiendo alguna de las tres técnicas señaladas anteriormente para la lectura de anillos en los otolitos. En la Figura 13 se representan gráficamente las longitudes teóricas calculadas para cada edad con estos parámetros. Tanto los parámetros como las curvas teóricas de crecimiento que se presentan están expresados en longitud a la horquilla, y se puede observar claramente las diferencias entre los tres parámetros y las curvas de crecimiento teórico resultantes tanto entre autores, como entre zonas y técnicas de preparación y lectura. 
Tabla 5. Parámetros de crecimiento en longitud a la horquilla de la ecuación de von Bertalanffy estimados por diversos autores a partir de la lectura de anillos anuales en otolitos de jurel T. murphyi proveniente de diversas zonas del Pacífico Sudoriental (adaptado de IFOP 1995).

Table 5. Growth parameters in fork length of the von Berlalanffy equation estimated by different authors, from reading annual rings in otoliths of Jack mackerel T. murphyi from various areas of the Southeast Pacific (adapted from IFOP 1995).

\begin{tabular}{|c|c|c|c|c|}
\hline Autores y tecnica de lectura & $\mathrm{L} \infty(\mathrm{cm})$ & k (anual) & $t_{0}$ (años) & Zona \\
\hline 1 Kaiser (1973)(a)(c) & 70.0800 & 0.1840 & -0.34070 & Antofagasta-Coquimbo-Valparaiso \\
\hline 2 Pavez \& Saa (1978)(c) & 108.2820 & 0.1088 & -0.87720 & Valparaiso \\
\hline 3 Aguayo et al (1981)(c) & 45.9000 & 0.1671 & -0.88170 & Arica-Iquique \\
\hline 4. Abramov \& Kotylar (1981)(a)(c) & 72.4700 & 0.0930 & -2.23280 & Perú \\
\hline 5. Dioses (1995)(c) & 79.1600 & 0.1500 & -0.28000 & Perú \\
\hline 6. Shevshuk \& Chur (1984)(a)(c) & 90.4100 & 0.1150 & -1.42900 & $8^{\circ}$ a $50^{\circ} \mathrm{S}$ \\
\hline 7. Castillo \& Arrizaga (1987)(c) & 65.2290 & 0.0741 & -2.41020 & Talcahuano \\
\hline 8. Shcherbithch (1991)(a)(c) & 91.2300 & 0.0910 & -1.58300 & - \\
\hline 9. Kochkin (1992)(c) & 74.2400 & 0.1109 & -0.81130 & $33^{\circ}$ a $39^{\circ} \mathrm{S}$ \\
\hline 10. Alegria et al. (1995)(b) & 78.4800 & 0.1540 & -0.09800 & Zona Norte de Chile \\
\hline 11. Alegria et al. (1995)(c) & 53.5000 & 0.1114 & -1.79680 & Zona Norte de Chile \\
\hline 12. Alegria et al. (1995)(d) & 48.4000 & 0.1840 & -0.94500 & Zona Norte de Chile \\
\hline
\end{tabular}

(a) Parametros estimados de los datos de los autores

(b) Lectura de incrementos diarios

(c) Lectura tradicional de anillos anuales

(d) Lectura de anillos anuales en cortes transversales

En cuanto a estas diferencias, se destaca que tanto Kaiser (1973) como Pavez y Saa (1978) trabajaron con muestras limitadas y con una cobertura insuficiente a uno y otro lado de los rangos de talla y edad. Es así que, al no contar con suficientes ejemplares de menor tamaño, ninguno de los dos autores detectó especímenes de edades 1,2 y 3, con lo que se aumenta la probabilidad de sobreestimar o subestimar la longitud asintótica $\mathrm{L}_{\infty}$, distorsionándose así los demás parámetros. Se observa también que en los parámetros

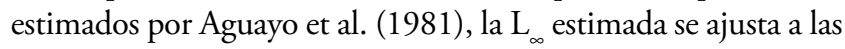
tallas máximas observadas en la pesquería industrial, pero no a las tallas máximas que se observan en la pesquería artesanal que son bastante más altas, sugiriendo una subestimación de $\mathrm{L}_{\infty}$ y una posible sobreestimación de $\mathrm{k}$ (dada la relación inversa entre $\mathrm{L}_{\infty} \mathrm{y}$ k) al usar muestras sólo de la pesca industrial.

Los otolitos de T. murphyi muestran una serie de anillos de crecimiento con zonas de crecimiento rápido (anillos opacos) alternadas a zonas de crecimiento lento (anillos hialinos). La formación de estos anillos dificulta la identificación del anillo

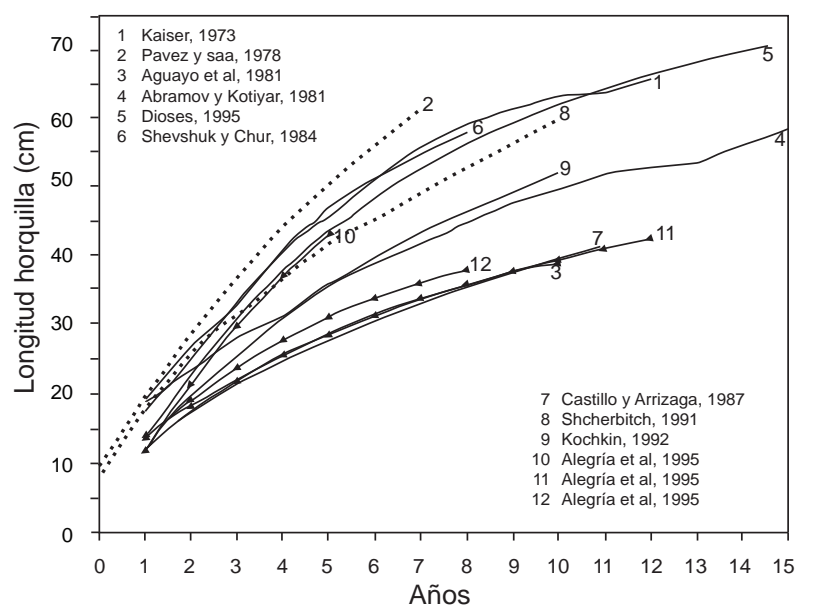

Figura 13. Curvas de crecimiento del jurel T. murphyi de distintas zonas del Pacífico Sudoriental según diversos autores (adaptado de IFOP 1995).

Figure 13. Growth curves of Jack mackerel T. murphyi for different zones of the Southeast Pacific according to various authors (adapted from IFOP 1995). anual y también la interpretación del borde marginal, el cual sólo se distingue si está completo y se le compara con zonas de crecimiento de ańos anteriores. Este hecho puede representar un problema particularmente difícil al determinar el primer anillo.

En las lecturas realizadas en el IMARPE se sigue el criterio de contar sólo los anillos completos, considerando como requisito que el anillo hialino debe tener un cierto grosor y una continuidad en el entorno de la mayor parte del otolito para diferenciarlo de los falsos anillos. La correcta observación de anillos completos es de suma importancia para diferenciar falsos anillos (o anillos incompletos) de los anillos anuales verdaderos. $\mathrm{Al}$ seguir este criterio se reduce la probabilidad de interpretar anillos secundarios o incompletos como anillos anuales, lo que introduciría sesgos en la confección de las claves talla-edad al sobreestimar la edad de los especímenes examinados.

Sin embargo, este mismo criterio de considerar como anuales sólo aquellos anillos hialinos ya completos precedidos de una zona o anillo opaco podría inducir a una sobreestimación de la edad de los individuos más jóvenes al asignar la edad 1 (primer anillo anual) a ejemplares de 6 a 11 meses de edad que ya habrían formado un primer anillo hialino completo precedido de un núcleo o primer anillo opaco. Como observan Perea et al. (2013), en el Perú el jurel T. murphyi tiene un periodo de desove relativamente amplio con un máximo en el mes de noviembre. Por otro lado, en este estudio se ha determinado (Fig. 8) que el anillo opaco se forma entre noviembre (primavera) y mayo (otoño) del año siguiente con un máximo entre marzo-abril (final del verano), mientras que el anillo hialino se forma entre junio y setiembre (invierno). De este modo, a partir de octubre los individuos nacidos el año anterior entre noviembre (durante el pico de máximo desove) y diciembre-febrero ya habrían tenido la oportunidad de formar un primer anillo hialino completo precedido un anillo o núcleo opaco. Al cumplir este requisito se estaría asignando la edad 1 a individuos que en realidad tendrían sólo entre 11 y 8 meses (dependiendo de si nacieron en noviembre o febrero). Este hecho explica la diferencia entre la longitud a la edad 1 calculada en este estudio y la longitud al año de edad 
(365 días) calculada por análisis de incrementos diarios por Goicochea et al. (2013).

El método basado en el análisis de la formación de los incrementos de crecimiento diarios permite validar los resultados obtenidos por el método tradicional de lectura de anillos anuales en los otolitos. Al respecto, Araya et al. (2003) demostraron que los microincrementos en los otolitos de T. murphyi corresponden a formaciones diarias.

En la Figura 13 se observa que las longitudes teóricas de la curva 10 de Alegría et al. (1995) calculadas en base a lectura de microincrementos para las edades 1 a 5 se aproximan a los valores de la curva 5, que corresponde a los resultados de la lectura directa de otolitos enteros de Dioses (1995) confirmados en este estudio. Estos resultados contribuirían a validar los parámetros estimados en el presente trabajo, por lo menos hasta la edad 5. Siempre con respecto a la Figura 13, es probable que en las lecturas y los cálculos de los parámetros de Aguayo et al. (1981) (curva 3), de Castillo y Arrizaga (1987) (curva 7), y de Alegría et al. (1985) por lectura rutinaria (curva 11) y por corte (curva 12), se estarían sobreestimando las edades. Como se puede apreciar, en las curvas 3, 7, 11 y 12 las longitudes teóricas corresponden a edades que son casi el doble que las mismas longitudes en las curvas 10 de Alegría et al. (1995), por incrementos diarios, y 5 de Dioses (1995), por lectura tradicional.

En Perú, Goicochea et al. (2013) han estudiado el periodo de formación del primer anillo de crecimiento en los otolitos, aplicando la misma técnica de análisis de microincrementos o lectura de anillos diarios aplicada por Alegría et al. (1995) para el T. murphyi de la zona norte de Chile. Con las lecturas de esta técnica y el ajuste del modelo de crecimiento de Laird-Gompertz se podría suponer que un individuo de un año de edad (365 días) tendría $195,3 \mathrm{~mm}$ de longitud total (17,7 cm de longitud a la horquilla).

Otro método que se emplea para validar las edades y las tasas de crecimiento estimadas por lecturas de otolitos es el que se basa en el seguimiento de una clase anual a través del tiempo mediante la progresión modal de frecuencia de tallas. Aplicando este método, Goicochea et al. (2013) encontraron que en 12 meses, entre enero 2011 y enero 2012, el crecimiento de los otolitos entre el segundo y el tercer anillo fue de $0,62 \mathrm{~mm}$ en promedio. En el presente trabajo se encontró que el incremento del segundo al tercer radio fue de $0,80 \mathrm{~mm}$. Sin embargo, a pesar de esta diferencia, los parámetros de crecimiento estimados por Goicochea et al. (2013) son similares a los estimados en este estudio.

Paralelamente, Díaz (2013) hace un análisis de las frecuencias de tallas del T. murphyi peruano y estima parámetros de crecimiento similares a los de este estudio.

El T. murphyi que habita en el mar peruano y aguas adyacentes presenta un crecimiento alométrico y diferente (mayor) a otros stocks o substocks de T. murphyi presentes en el Pacífico Sudoriental y que ocupan espacios más australes y en algunos casos muchos más alejados de la costa sudamericana. Según los parámetros estimados, el jurel T. murphyi peruano tendría una velocidad de crecimiento mayor que el jurel T. murphyi de zonas más australes.

\section{Literatura citada}

Abramov A.A \& A.N. Kotlyar . 1980. Some Biological Features of the Peruvian Jack Mackerel, Trachurus symmetricus murphyi. J. Ichthyol. 20(1) 25-31.
Aguayo M., A. Estay \& V. Ojeda 1981. Determinación de edad y crecimiento del jurel (Trachurus murphyi) en las zonas Arica-Coquimbo y Talcahuano. Informe Subsecretaría de Pesca. Inst. Fom. Pesq. Santiago, Chile, 88p.

Alegría V., J. Oliva, H. Robotham et al. 1995. Estudio biológico pesquero sobre el recurso jurel en la zona norte de Chile (Regiones I y II). Informe Final, Proyecto FIP 93-18. Fondo de Investigación Pesquera. Instituto de Fomento Pesquero, Chile: 221 pp.

Araya M., M. Medina \& H. Arancibia . 2003. Preliminary results of the empirical validation of daily increments in otoliths of Jack mackerel Trachurus symetricus (Aires, 1855) marked with oxytetracycline. Scientia marina 67 (4): 471-475.

Castillo G. \& A. Arizaga. 1987. Edad y crecimiento del jurel Trachurus symmetricus murphyi (Nichols, 1920) en aguas de Chile Central. Biología Pesquera. Pont. Univ. Católica de Chile (Talcahuano) 16: 19-33.

Díaz E. 2013. Estimación del crecimiento del jurel (Trachurus murphyi) capturado en el Perú a partir del análisis de las frecuencias por tallas. En: Csirke J., R. Guevara-Carrasco \& M. Espino (Eds.). Ecología, pesquería y conservación del jurel (Trachurus murphyi) en el Perú. Rev. peru. biol. número especial 20(1): 061 - 066

Dioses T. 1985. Edad y crecimiento de la merluza Merluccius gayi peruanus (G). Anales I Congreso Nacional de Biología Pesquera. Alvaro E. Tresierra Aguilar (editor). Colegio de Biólogos Regional del Norte. Trujillo, Perú . pp. 65-69.

Dioses T. 1995. Análisis de la distribución y abundancia de los recursos jurel y caballa frente a la costa peruana. Inf. Prog. Inst. Mar Perú-Callao (3): 65-69 (mimeo).

Ford, E. 1933. An account of the herring investigation conducted at Plymouth during the years from 1924-1933. J. Mar. Biol. Assoc., 19: 305-384.

Geldenhuys N.D. 1973. Growth of the South African Maasbanker Trachurus trachurus L. and age composition of the catches, 1950-1971. Rep. of S. African Dept. Ind., Sea Fish. Branch Invest. Rep. 101, 1-24

Goicochea C., J. Mostacero, P. Moquillaza, T. Dioses, Y. Topiño \& Renato Guevara-Carrasco. 2013. Validación de los anillos de crecimiento de otolitos del jurel Trachurus murphyi. En: Csirke J., R. GuevaraCarrasco \& M. Espino (Eds.). Ecología, pesquería y conservación del jurel (Trachurus murphyi) en el Perú. Rev. peru. biol. número especial 20(1): $053-060$

Gulland J.A. (Ed.) 1977. Fish population dynamics. London. J.Wiley and Sons pp. 372

IFOP. 1995. Estudio biológico pesquero sobre el recurso jurel en la zona norte (Regiones I y II). Informes Técnicos FIP, Informe Final Proyecto FIP-IT/93-17. IFOP, enero 1995: 374 pp.

Kaiser C.E. 1973. Age and growth of Horse Mackerel Trachurus murphyi of Chile. Trans. Ame. Fish. Soc. 102 (3): 591-595.

Kochkin P.N. 1994. Age Determination and Estimate of Growth Rate for the Peruvian Jack Jack Mackerel, Trachurus symmetricus murphyi. J. of Ichthyology 34 (3):39-50 .

Misu H. \& S. Hamasaki. 1971. Age and growth of Peruvian hake Merluccius gayi (Guichenot). Bull. Saikai Reg. Fish. Res. Lab. (41):93-106 .

Pavez C.P. \& C.E. Saa. 1978. Determinación de edad y crecimiento del jurel Trachurus murphyi Nichols, 1920. Inv. Mar. Valparaíso, Chile. Vol. 6 (6): 89-100.

Perea A., J. Mori, B. Buitrón \& J. Sánchez. 2013. Aspectos reproductivos del jurel Trachurus murphyien el Perú. En: Csirke J., R. Guevara-Carrasco \& M. Espino (Eds.). Ecología, pesquería y conservación del jurel (Trachurus murphyi) en el Perú. Rev. peru. biol. número especial 20(1): $029-034$

Samamé M. 1977. Determinación de la edad y crecimiento de la sardina Sardinops sagax sagax (J.). Bol. Inst. Mar Perú - Callao, 3 (3): 95-112 .

Shcherbitch, L. V. 1991. Fishery-ecological researches in the South Pacific Ocean. Trudy AtlantNIRO:35-53 (en ruso)

Shevchuk L.V. \& V.N. Chur . 1984. Métodos de determinación de la edad del jurel (Trachurus murphyi N.) del Pacífico Suroriental. En: Investigaciones pesqueras $\mathrm{d}$ las regiones abiertas del Océano Mundial. VNIRO, Moscú : 97-105 (en ruso).

Walford, L.A. 1946. A New graphic method of describing the growth of animals. Bio. Bull. 90(2): 141-147. 